\title{
Functional impression and cast construction technique for implant retained auricular prosthesis
}

\author{
Ajay Jain ${ }^{1 *}$, Akshaya Bhargava ${ }^{2}$ and Ugrappa Sridevi ${ }^{3}$ \\ ${ }^{1}$ Senior Lecturer, Department of Prosthodontics, Faculty of Dentistry, AIMST Dental Institute, AIMST University, Kedah, Malaysia \\ ${ }^{2}$ Director-Principal and Head of the Department, SGT Dental college, Gurgaon, Haryana, India \\ ${ }^{3}$ Lecturer, Department of Oral Medicine \& Radiology, Faculty of Dentistry, AIMST Dental Institute, AIMST University, Kedah, Malaysia
}

\begin{abstract}
Implant-retained auricular prosthesis presents a difficult task in relation to the esthetic placement of the margins of the prosthesis. A technique for the functional impression and master cast fabrication to fabricate an implant-retained auricular prosthesis is described. This technique allows esthetic placement of the margin, fabrication of imperishable master cast with pre-planned block-out and incorporation of a replica of the retentive bar, which allows processing of the prosthesis without returning the retentive bar to the mold.
\end{abstract}

\section{Introduction}

Esthetic placement of the margin of implant retained auricular prosthesis pose a great challenge for the maxillofacial prosthodontist. Functional movement of the mandible and changes in head posture [1] tends to lift up the margin of the prosthesis away from the underlying skin. To maintain the marginal integrity of the prosthesis during function, the impression must be made in functional state by using custom tray. The goal of this technique to fabricate the prosthesis, which is in functional form, helps to retain the marginal integrity and construction of model without damage to the retentive bar and clip assembly.

\section{Procedure}

1. After blocked out the undercuts (Figure 1), irreversible hydrocolloid alginate impression (Neocolloid, Zhermack, Italy) was made (Figure 2) and cast it with the type IV dental stone (Ultrarock, Kalabhai, India).

2. Clip retained acrylic substructure was fabricated with

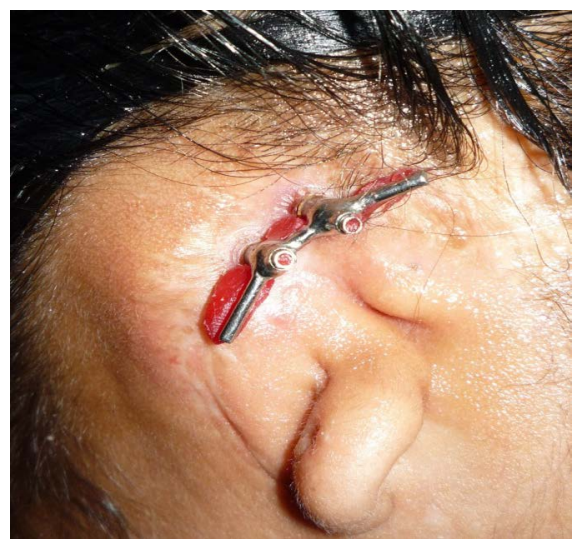

Figure 1. Block out of undercuts. orientation groove and adapted along with the wax spacer (Figure 3) over the diagnostic cast and custom tray was fabricated using autopolymerizing acrylic resin (Shanghai Pigeon Dental Mfg. Co., Ltd., China) (Figure 4).

3. It can be easily appreciated that custom tray fabricated having orientation notches which will fit exactly over the grooved acrylic substructure (Figure 5).

4. Acrylic substructure was placed over the bar and functional impression was made by asking the patient to depress

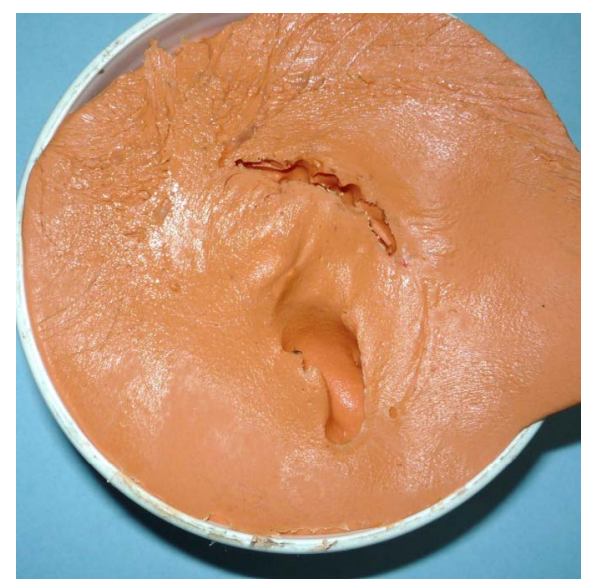

Figure 2. Diagnostic impression using alginate impression material.

Correspondence to: Dr. Ajay Jain, Senior Lecturer, Faculty of Dentistry, AIMST Dental Institute, AIMST University, Kedah, Malaysia, Tel: +60109454204, E-mail: jainajay9@gmail.com

Key words: auricular prosthesis, functional impression, implant, master cast Received: May 12, 2016; Accepted: June 24, 2016; Published: June 29, 2016 


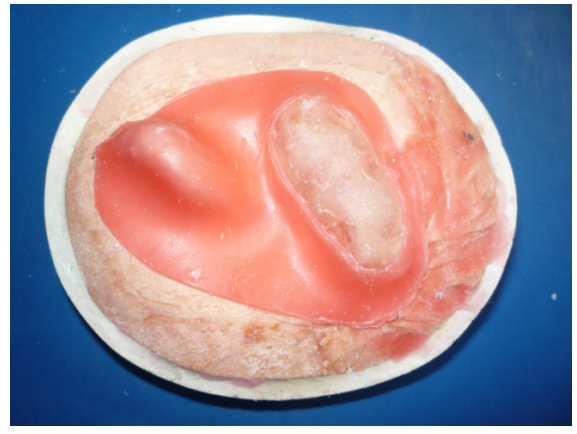

Figure 3. Acrylic substructure with wax spacer.

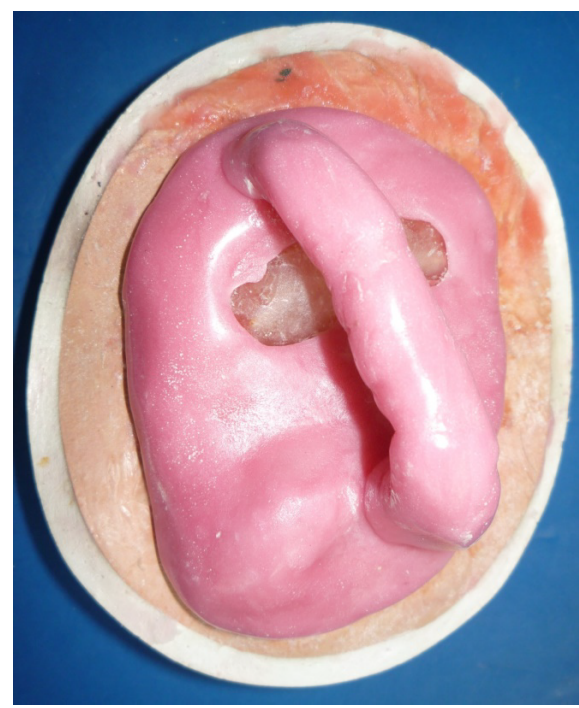

Figure 4. Custom tray.

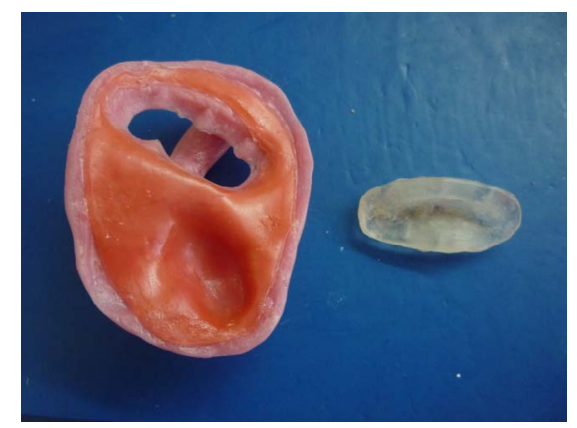

Figure 5. Orientation of custom tray over acrylic substructure.

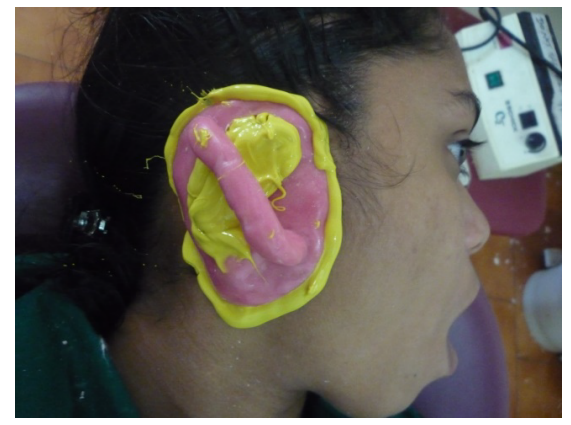

Figure 6. Making of functional impression. her mandible and move right and left (Figure 6), using polyvinylsiloxane light body elastomeric impression material (Aquasil Ultra LV, Dentsply).

5. Final impression obtained (Figure 7) and evaluated for the accuracy and polyvinylsiloxane putty elastomeric impression material was pushed into the region of bar and clip assembly to replicate the same, and with stapler pins to achieve mechanical retention in the master cast (Figure 8).

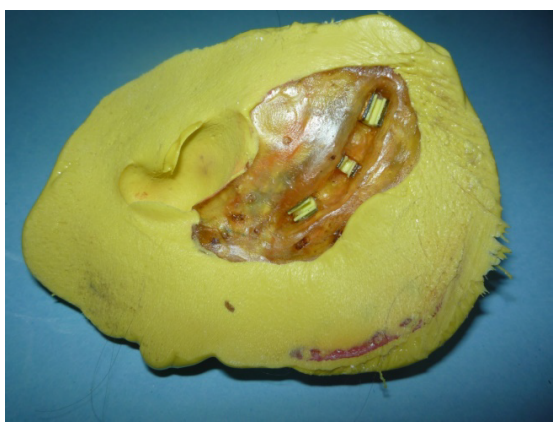

Figure 7. Final impression.

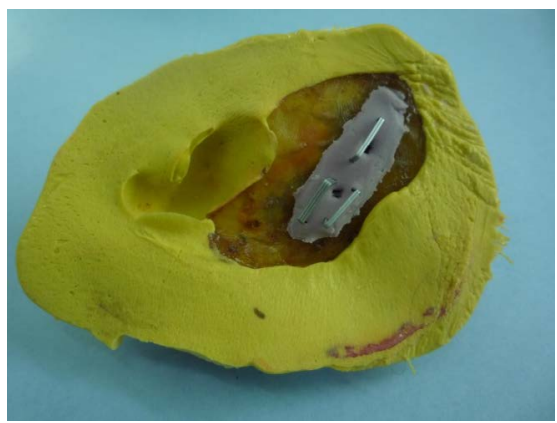

Figure 8. Bar and clip assembly replicated.

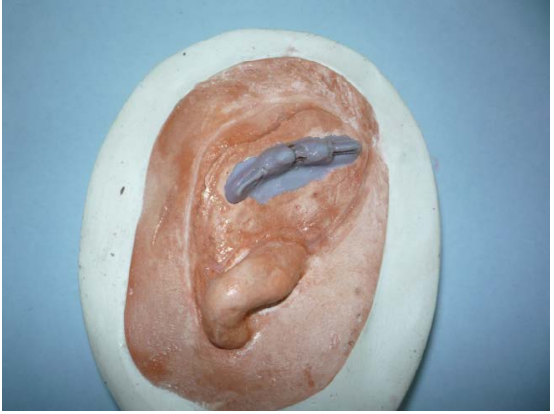

Figure 9. Master cast.

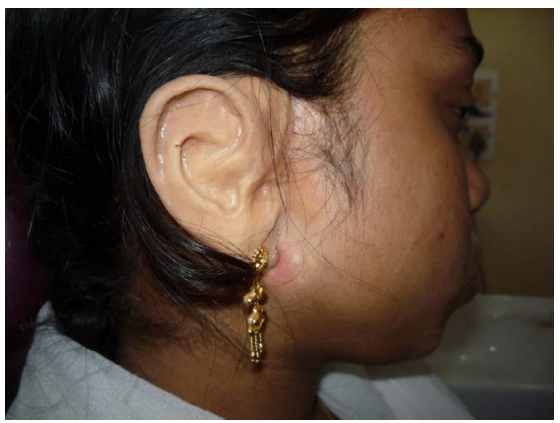

Figure 10. Delivery of the prosthesis. 
6. Master cast obtained which replicates the bar and clip assembly (Figure 9) and there was no need for it to transfer from the patient and then fabrication of prosthesis was performed in usual manner using this master cast.

7. The prosthesis delivered to the patient that was highly esthetic in terms of positioning of anterior margin during functional movement (Figure 10).

\section{Discussion}

A plethora of technique has been described in the literature for making impression of implant retained auricular prosthesis. As described by Thomas [2], add stone or wax to the surface of the master cast to create space and the anterior margin is usually managed by scraping the master cast and then smoothing the area with abrasive paper, but this technique is unpredictable. Indirect techniques $[3,4]$ described in the literature require that the retentive bar be returned to the mold for processing. This is inconvenient for the patient and risks damage to the bar. The present technique described overcome all these problems and allows practitioner to provide esthetic anterior margin placement for implant retained auricular prosthesis.

\section{References}

1. Wolfaardt JF, Coss P (1996) An impression and cast construction technique for implantretained auricular prostheses. J Prosthet Dent 75: 45-49. [Crossref]

2. Thomas KF (1994) Prosthetic rehabilitation. London: Quintessence Pub1 Co: 69-98.

3. Bergstrom K (1993) Prosthetic techniques for orbital defects. Bone anchored applications. In: Williams E, Nobelpharma International Updates. Nobelpharma, Goteborg 2: 5-8.

4. Tjellstrom A, Jansson K, Brånemark PI (1992) Craniofacial defects. In: Worthington $\mathrm{P}$, Brånemark PI, editors. Advanced Osseointegration Surgery Applications in the Maxillofacial Region. Chicago, Ill, USA: Quintessence: 293-312.

Copyright: (2016 Jain A. This is an open-access article distributed under the terms of the Creative Commons Attribution License, which permits unrestricted use, distribution, and reproduction in any medium, provided the original author and source are credited. 